\title{
Carcinoma de osso temporal sem foco metastático primário: relato de caso e revisão de literatura
}

\author{
Marcelo Lemos Vieira da Cunha', Clézio Alex Onuki Castro', \\ Matheus Lemos Vieira da Cunha², Daiana Paola Perin ${ }^{2}$ \\ Departamento de Neurocirurgia do Hospital Regional do Oeste, Chapecó, SC, Brasil.
}

\section{RESUMO}

Os tumores do osso temporal são raros e geralmente apresentam sintomas como otorreia, otalgia e hipoacusia, por isso podem ser facilmente confundidos com um processo infeccioso, retardando o diagnóstico e piorando o prognóstico do paciente. KS, 7 anos, masculino. Estado geral: regular. Ao exame: consciente; linfonodos cervicais palpáveis, móveis; massa palpável, imóvel e indolor em topografia retroauricular esquerda; surdez à esquerda e paralisia facial esquerda. Tomografia computadorizada de crânio evidenciou lesão expansiva do osso temporal captante de contraste com extensão para fossa média e posterior do crânio. Realizada complementação radiológica com ressonância de encéfalo, a qual apresentou imagem hipercaptante em T1 contrastado. Nas incidências T2, observa-se edema lobotemporal adjacente à lesão. Exame angiográfico cerebral apresentou obstrução tumoral do seio sigmoide esquerdo. Paciente submetido à mastoidectomia radical esquerda com ligadura e ressecção do seio sigmoide esquerdo resultando em ressecção completa lesional. Anatomopatológico e exame imunoistoquímico compatíveis com adenocarcinoma. Encaminhado para terapia oncológica com quimioterapia e radioterapia. Óbito após quatro meses do tratamento neurocirúrgico. O diagnóstico precoce associado com a extensão do tumor acarreta melhor ou pior prognóstico para os pacientes acometidos por essa moléstia.

\section{PALAVRAS-CHAVE}

Osso temporal, carcinoma, neoplasias cranianas, otite.

\section{ABSTRACT}

Carcinoma temporal bone unfocused metastatic primary: case report and literature review Tumors of the temporal bone are rare and usually have symptoms such as otorrhea, otalgia and hearing loss and, therefore, can be easily confused with an infectious process delaying diagnosis and worse prognosis. KS, age 7, male. General condition: regular. On examination: conscious, palpable cervical lymph nodes, mobile; palpable mass, painless and property surveying retroauricular left, left deafness and facial paralysis left. Cranial computed tomography showed a lesion of the temporal bone with extension to the middle fossa and posterior skull that enhance with contrast. Performed complementation with radiological MRI brain image which showed uptake, in contrast T1. Incidences in T2, there is edema temporal lobe adjacent to the lesion. Cerebral angiography showed a tumor obstructing the left sigmoid sinus. Patient underwent left radical mastoidectomy with ligation and resection of the left sigmoid sinus resulting in complete lesional resection. Histopathological and immunohistochemical examination compatible with adenocarcinoma. Referred for cancer therapy with chemotherapy and radiation. Death within four months after neurosurgical treatment. Early diagnosis associated with tumor extension carries a better or worse prognosis for patients affected by this disease.

\section{KEYWORDS}

Temporal bone, carcinoma, skull neoplasms, otitis.

1 Neurocirurgião do Hospital Regional do Oeste, Chapecó, SC, Brasil.

2 Acadêmico(a) do curso de Medicina da Universidade do Vale do Itajaí (Univali), Itajaí, SC, Brasil. 


\section{Introdução}

Os tumores do osso temporal são raros e podem ser histologicamente benignos (adenoma, papiloma invertido, meningioma) ou malignos. Lesões malignas podem ter origem no epitélio glandular, mesenquimal ou salivar e incluem tumor primário (carcinoma de células escamosas, adenocarcinoma), metastático (com origem em mama, pulmão, rim, estômago, laringe) e secundário (carcinoma basocelular, carcinoma espinocelular, outros carcinomas e sarcomas). Oitenta e seis por cento desses tumores são carcinoma espinocelular (CEC) ${ }^{1,2}$ Apesar disso, o carcinoma espinocelular de osso temporal é responsável por menos de $0,2 \%$ dos tumores de cabeça e pescoço e está associado a desfecho desfavorável. ${ }^{3}$ São considerados fatores de pior prognóstico linfonodopatia regional, carcinoma de células escamosas, paralisia do nervo facial moderada e severa e margens cirúrgicas positivas. ${ }^{4}$

Pelo fato de as manifestações clínicas serem frequentemente inespecíficas e a progressão da doença ser rápida, geralmente o carcinoma é diagnosticado em estágio avançado, com limitado sucesso terapêutico. Frequentemente, sintomas corriqueiros como otorreia, otalgia e hipoacusia podem ser erroneamente diagnosticados como processos infecciosos, retardando o diagnóstico e piorando o prognóstico do paciente. ${ }^{4}$

Segundo Noorizan e Asma, ${ }^{1}$ otorreia crônica e inflamação ou colesteatoma do conduto auditivo externo (CAE) e do ouvido médio são fatores de risco associados ao desenvolvimento de tumor, por levarem à metaplasia escamosa. Se a massa se estender até o ouvido interno e o osso petroso, o paciente desenvolverá vestibulopatia, neuropatias cranianas (nervo comumente acometido: facial), otorreia ou cefaleia. Se o tumor continuar a se espalhar na fossa média e posterior, até a região petroclival ou seio cavernoso, o prognóstico passa a ser sombrio.

São padrões de invasão do osso temporal as erosões nas seguintes direções: superiormente através do tímpano na fossa craniana média, anterior à fossa glenoide e ao espaço infratemporal; inferiormente através do forame jugular; posteriormente nas células aéreas da mastoide; medialmente ao canal carotídeo e, muito raramente, através da cápsula ótica. As invasões intracerebral e da base do crânio são mais frequentes e mais importantes. ${ }^{2}$

A detecção precoce do tumor no osso temporal se baseia em análise histopatológica de tecidos removidos do canal externo e do ouvido médio. A tomografia computadorizada delineia precisamente a extensão óssea do tumor e melhora o planejamento da terapia. ${ }^{5}$ A ressonância magnética (RM) pode ser usada para diagnóstico adjuvante na visualização de alterações em partes moles extratemporais e na disseminação do tumor ao longo dos canais vasculares e das vias neurais, bem como na extensão intracraniana. ${ }^{6}$

O tratamento cirúrgico das neoplasias malignas do osso temporal é estritamente dependente da delimitação radiográfica, da extensão da doença e da relação do tumor com estruturas neurovasculares adjacentes. ${ }^{5}$ O tratamento curativo padrão para carcinoma de osso temporal é en bloc excisão com margens livres, que inclui ressecção do osso temporal lateral ou ressecção subtotal do osso temporal - para tumores se estendendo além da membrana timpânica. A necessidade de cirurgias mais extensas deve ser discutida individualmente com o paciente e traz alta morbidade. No pós-operatório, radioterapia com ou sem quimioterapia é geralmente usada como adjuvante em pacientes com doença extensa. ${ }^{4}$

\section{Relato do caso}

Paciente masculino, 7 anos de idade, encaminhado do Hospital da Criança de Chapecó por quadro de paralisia facial periférica esquerda com instalação sábita há uma semana. À sua admissão no serviço de neurocirurgia do Hospital Regional do Oeste (HRO) em Chapecó/ SC, apresentava-se em regular estado geral, com perda ponderal estimada pelos pais de cerca de $6 \mathrm{~kg}$ no último trimestre. Em anamnese dirigida, familiares relatam inúmeros tratamentos para otite com otorreia em postos de saúde nos últimos quatro meses, sem obter controle da doença. Ao exame, estava consciente e apresentava linfonodos cervicais palpáveis, móveis, indolores, com aproximadamente $4 \times 3 \mathrm{~cm}$, e massa palpável, imóvel e indolor em topografia retroauricular esquerda. Ao exame neurológico: força motora grau $\mathrm{V}$ em membros superiores e inferiores; ausência de alteração às provas cerebelares, meningorradiculares, sensibilidade ou de reflexos profundos. Ao exame dos pares cranianos, verificaram-se surdez à esquerda e paralisia facial esquerda (House-Breckman: V).

À tomografia computadorizada de crânio, evidenciou-se lesão expansiva do osso temporal captante de contraste com extensão para fossa média e posterior do crânio (Figura 1). Realizada complementação radiológica com ressonância de encéfalo, a qual apresentou imagem hipercaptante em $\mathrm{T} 1$ contrastado com edema perilesional (Figuras 2 e 3 ).

Investigação de foco primário não identificou outras lesões em TC de tórax, abdome, pescoço e pelve.

Exame angiográfico cerebral apresentou obstrução tumoral do seio sigmoide esquerdo. Paciente submetido à mastoidectomia radical esquerda com ligadura e ressecção do seio sigmoide esquerdo, resultando em ressecção completa lesional (Figura 4). 


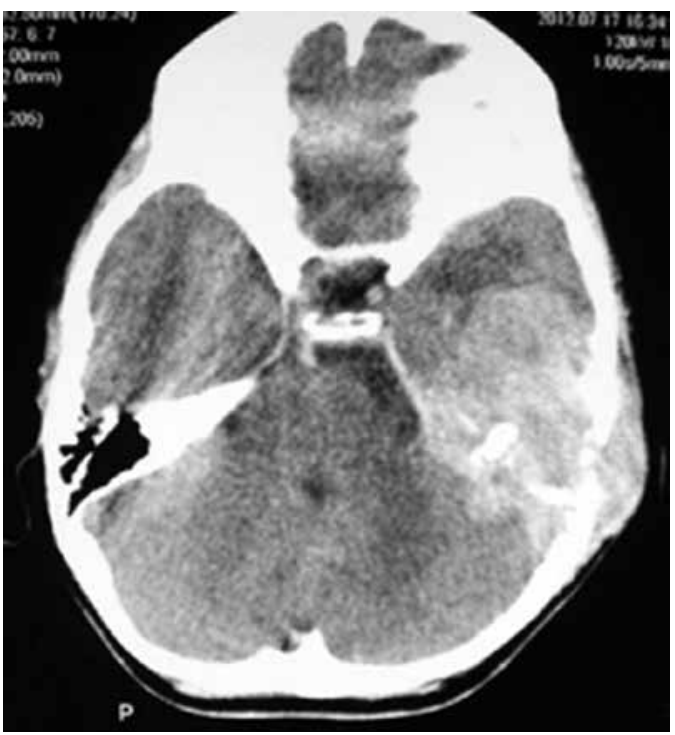

Figura 1 - TC de crânio pré-operatória em corte axial demonstrando lesão expansiva hipercaptante em osso petroso esquerdo.

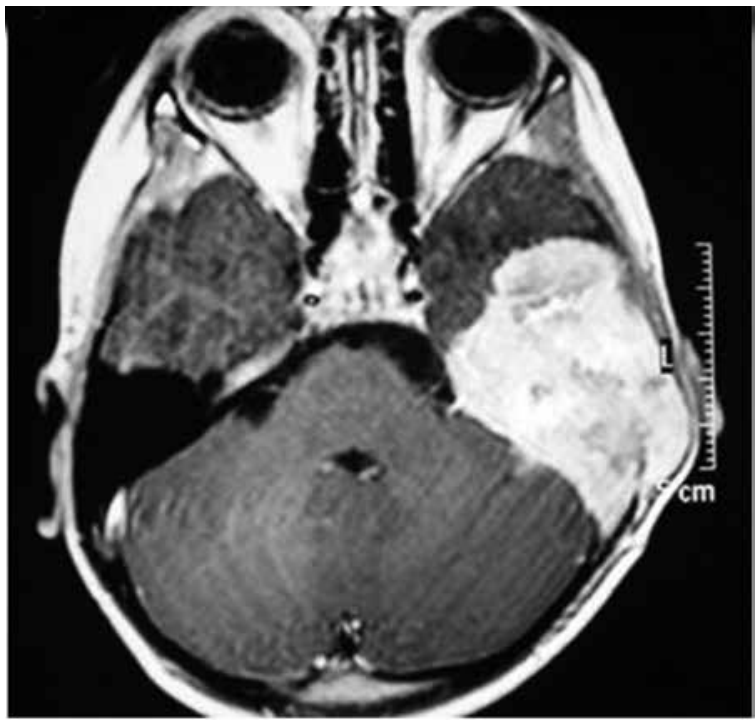

Figura 2 - RM de encéfalo T1 contrastada em corte axial, evidenciando processo tumoral em pirâmide petrosa esquerda com extensão à fossa média e posterior.

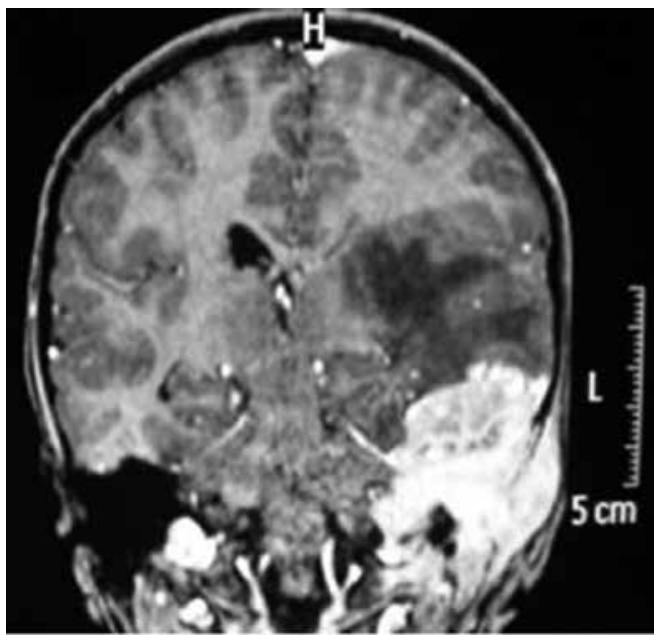

Figura 3 - RM de encéfalo T1 contrastada em corte coronal da referida lesão.

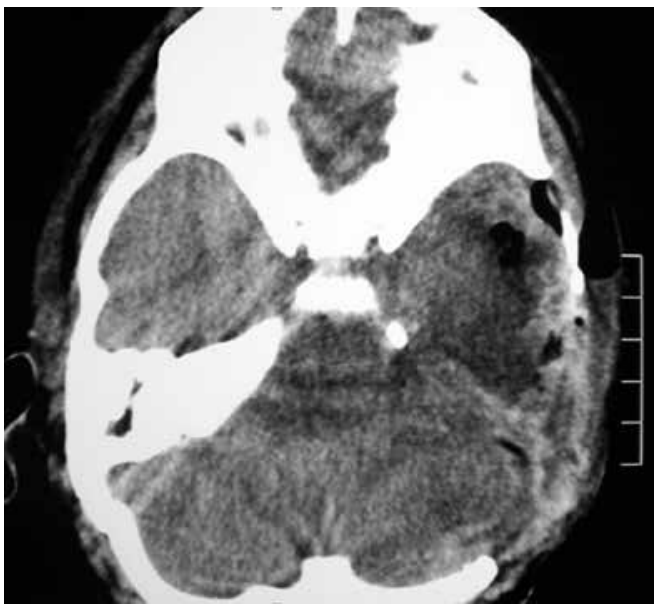

Figura 4 - TC de crânio contrastada após tratamento neurocirúrgico com ressecção total da lesão.

Anatomopatológico e exame imunoistoquímico compatíveis com adenocarcinoma glandular focal (Figura 5). Encaminhado para terapia oncológica com quimioterapia e radioterapia. Óbito após quatro meses do tratamento neurocirúrgico.
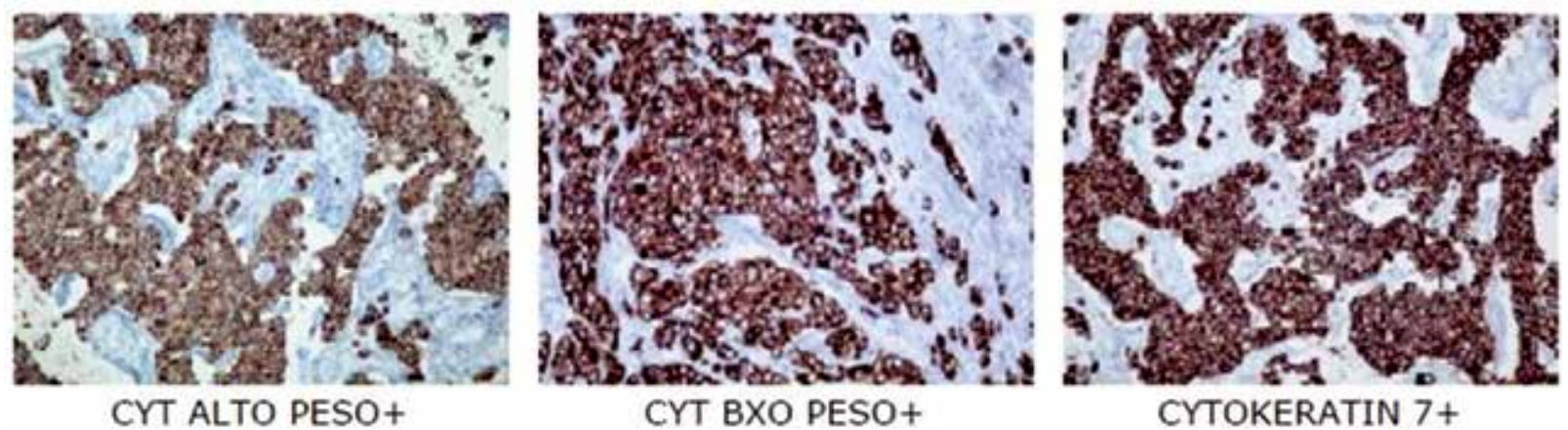

Figura 5 - Imunoistoquímica com diagnóstico de adenocarcinoma glandular focal. 


\section{Discussão}

Em muitos casos, a distinção entre o carcinoma de osso temporal e um processo infeccioso como otite média crônica com ou sem colesteatoma é difícil. Isso porque os sintomas de otorreia, dor e hipoacusia são comuns, seguidos de surdez, zumbido, paralisia do nervo facial e vertigem. ${ }^{4}$

O diagnóstico pode ser facilmente despercebido pelo fato de os sintomas serem confundidos com os de uma infecção. ${ }^{1}$ Com isso, ocorre atraso para realizar o diagnóstico definitivo, interferindo diretamente no prognóstico do paciente. É necessário atentar ao fato de que sintomas comuns como otorreia crônica ou inflamação e colesteatoma podem ser predisponentes à metaplasia escamosa, ${ }^{1}$ precursora de carcinoma.

Adenopatia é uma queixa frequente nos consultórios pediátricos que, na maioria das vezes, corresponde a um processo benigno. A indicação precoce de biópsia é controversa, uma vez que se trata de um procedimento invasivo que muitas vezes necessita de anestesia. Em contrapartida, sabe-se que a presença de doença linfonodal no carcinoma do osso temporal, por exemplo, é um significativo fator de pior prognóstico. Qualquer envolvimento linfonodal de caracteríticas malignas deve, portanto, ser sinal de alerta para doença avançada. ${ }^{6}$

Como exame auxiliar na avaliação, pode-se utilizar a tomografia computadorizada de crânio - ou mastoide - que no caso do nosso paciente revelou lesão petrosa erosiva óssea com invasão dural subjacente. Porém, os achados tomográficos do carcinoma de células escamosas são iguais aos achados dos adenocarcinomas, o que não permite diferenciá-los. No entanto, a análise conjunta da clínica do paciente e do exame tomográfico auxilia na diferenciação entre eles, já que presença de metástase para linfonodos é mais sugestiva de adenocarcinomas. Para avaliação de otite média crônica, a tomografia computadorizada de alta resolução é o exame de maior custo-benefício, devido à informação anatômica proporcionada.?

O tratamento otimizado de neoplasias malignas do osso temporal permanece obscuro por conta de um contínuo debate no tocante ao estadiamento, à utilidade da avaliação radiográfica pré-operatória, à extensão e nomenclatura dos procedimentos cirúrgicos e ao uso de radiação adjuvante. ${ }^{3}$ Não há estudos randomizados que relatem neoplasias primárias e secundárias de osso temporal. Em geral, todos os estudos são séries de casos. ${ }^{2} \mathrm{O}$ número limitado de casos de neoplasias malignas do osso temporal impede que cheguemos a conclusões definitivas quanto a um protocolo otimizado de tratamento. ${ }^{3}$ A ressecção total do osso temporal, combinada com radioterapia pós-operatória, parece estar associada com melhora da sobrevida. No entanto, esse tipo de tratamento pode não ser aplicável a alguns pacientes, nos quais a remoção total apresenta riscos aumentados para ocorrer fístula liquórica ou de haver propagação endocraniana. ${ }^{2}$ Atualmente, tem-se como tratamento-padrão a excisão em bloco com margens livres. ${ }^{4}$

Sabe-se que a extensão para ouvido médio do adenocarcinoma primário, como no caso do paciente em questão, é seguida por maior propagação dentro do osso temporal e estruturas vitais adjacentes, por isso há poucas diferenças na sobrevivência em tal fase, independente do tipo de tumor ou do tipo de procedimento cirúrgico. ${ }^{5}$

Segundo Stankovic, ${ }^{2}$ a adição de terapia de radiação para o tratamento cirúrgico dos tumores possui relação com a área acometida. Se o tumor se limita ao canal auditivo externo, não houve aumento da sobrevida dos pacientes, independentemente da técnica cirúrgica empregada -mastoidectomia radical ou subtotal. Já quando o tumor se estende para o ouvido médio, os resultados demonstraram melhorar a sobrevida daqueles que associaram a terapia de radiação com a cirurgia para ressecção tumoral ampla, porém o valor exato da taxa de sobrevida ainda é incerto.

Atualmente não existe nenhum sistema de estadiamento universalmente aceito para o tratamento de carcinoma de osso temporal devido a sua raridade. ${ }^{8}$

Madsen et al., ${ }^{9}$ pelo fato de a doença possuir baixa incidência, sugere criar centros especializados na Dinamarca, com equipes multidisciplinares, para melhorar os resultados relativos a essa moléstia.

Blair e Birch ${ }^{10}$ afirmam que estudos dos padrões de incidência de doença neoplásica são importantes, pois podem fornecer uma indicação de que os níveis de carcinógenos ambientais estão mudando e identificar fatores etiológicos, o que poderia levar ao desenvolvimento de medidas preventivas e fornecer informações para planejamento em saúde.

Pelo fato de o carcinoma de osso temporal ser bastante raro, ainda existem poucos relatos de casos sobre o tema. Talvez seja por isso que ainda não possuímos um tratamento a ser seguido de forma uniforme. A única certeza de que dispomos é que o momento do diagnóstico associado à extensão do tumor acarreta melhor ou pior prognóstico para os pacientes acometidos por essa enfermidade.

\section{Conflito de interesses}

Os autores declaram não haver conflito de interesses. 


\section{Referências}

1. Noorizan Y, Asma A. Temporal bone carcinoma: a case report. Med J Malaysia. 2010;65(2):162-4.

2. Stankovic M. Temporal bone carcinoma: results of surgery for primary and secondary malignancies. Mediterr $\mathrm{J}$ Otol. 2005;1:1-7.

3. Arora S, Sharma JK, Pippal S, Sethi Y, Yadav A. Temporal bone carcinoma with intracranial extension. Braz J Otorhinolaryngol. 2009;75(5):765.

4. Rusydi WZ, Goh BS. Primary undifferentiated carcinoma of the temporal bone: a new entity? Rawal Med J. 2013;38(1):86-9.

5. Stankovic M. Carcinoma of temporal bone: outcome of surgical therapy depending on stage and type of tumor. Arch Oncol. 2004;12(Suppl 1):7.

6. Ong CK, Pua U, Chong VF. Imaging of carcinoma of the external auditory canal: a pictorial essay. Cancer Imaging. 2008;8:191-8.
7. Prata AA, Antunes ML, Abreu CE, Frazatto R, Lima BT. Estudo comparativo entre achados radiológicos e cirúrgicos na otite média crônica. Arquivos Int Otorrinolaringol (Impr). 2011;15(1):72-8.

8. Lim LH, Goh YH, Chan YM, Chong VF, Low WK. Malignancy of the temporal bone and external auditory canal. Otolaryngol Head Neck Surg. 2000;122(6):882-6.

9. Madsen AR, Gundgaard MG, Hoff CM, Maare C, Holmboe P, Knap M, et al. Cancer of the external auditory canal and middle ear in Denmark from 1992 to 2001 . Head Neck. 2008;30(10):1332-8.

10. Blair V, Birch JM. Patterns and temporal trends in the incidence of malignant disease in children: II. Solid tumours of childhood. Eur J Cancer. 1994;30A(10):1498-511.

Endereço para correspondência

Marcelo Lemos Vieira da Cunha

Rua Rui Barbosa, 93 E, ap. 501, Centro

89801-040 - Chapecó, SC, Brasil

E-mail: marcelolvc@yahoo.com.br 